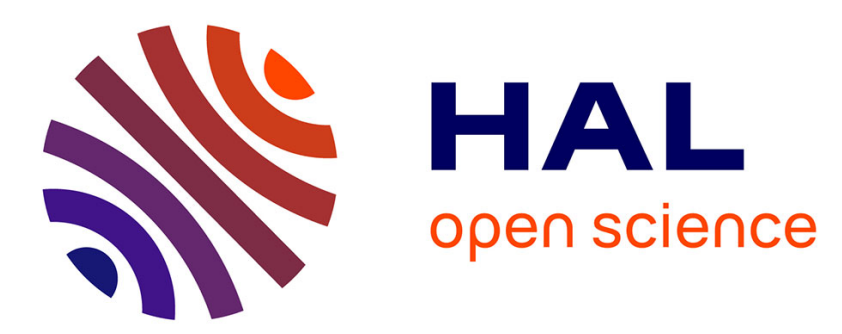

\title{
Sur quelques propriétés thermodynamiques d'une phase solide du gallium instable à la pression atmosphérique
}

\author{
A. Defrain, Israël Epelboin
}

\section{To cite this version:}

A. Defrain, Israël Epelboin. Sur quelques propriétés thermodynamiques d'une phase solide du gallium instable à la pression atmosphérique. J. Phys. Radium, 1960, 21 (1), pp.76-77. 10.1051/jphysrad:0196000210107601 . jpa-00236196

\section{HAL Id: jpa-00236196 https://hal.science/jpa-00236196}

Submitted on 1 Jan 1960

HAL is a multi-disciplinary open access archive for the deposit and dissemination of scientific research documents, whether they are published or not. The documents may come from teaching and research institutions in France or abroad, or from public or private research centers.
L'archive ouverte pluridisciplinaire HAL, est destinée au dépôt et à la diffusion de documents scientifiques de niveau recherche, publiés ou non, émanant des établissements d'enseignement et de recherche français ou étrangers, des laboratoires publics ou privés. 


\section{SUR QUELQUES PROPRIÉTÉS}

THERMODYNAMIQUES

D 'UNE PHASE SOLIDE DU GALLIUM INSTABLE

A LA PRESSION ATMOSPHÉRIQUE

Par A. Defrain et I. Epelboin,

Laboratoire de Physique (Enseignement) de la Faculté des Sciences de Paris.

En se basant sur le diagramme pression-température du gallium établi par Bridgman en 1935 [1], on peut prévoir l'existence d'un équilibre métastable entre la phase II solide stable aux pressions supérieures à

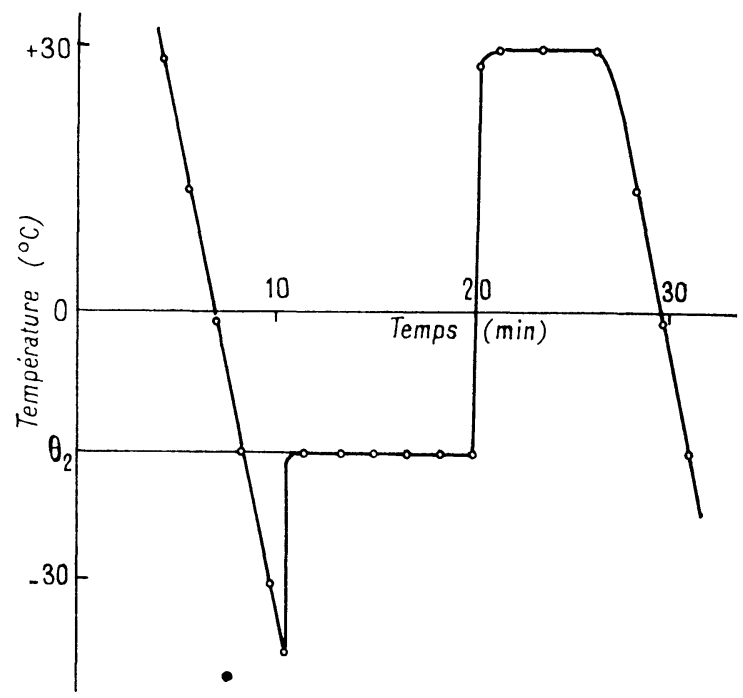

Fir. 1.

$12050 \mathrm{~kg} / \mathrm{cm}^{2}$ et l'état liquide ; une extrapolation linéaire de la courbe d'équilibre gallium II-liquide jusqu'à la pression atmosphérique conduit à une température d'équilibre de $-15,8^{\circ} \mathrm{C}$.

Le décapage préalable du métal dans une solution bouillante d'acide chlorhydrique nous permet de le maintenir en surfusion jusqu'à -40 o $\mathrm{C}$. Suivant la 
pureté du métal, nous obtenons deux types de courbe de refroidissement. Pour un métal comprenant plus de $99,99 \%$ de gallium, la courbe obtenue est représentée sur la figure 1 ; si le taux d'impuretés est de l'ordre de $0,1 \%$, la courbe représentative est donnée sur la figure 2 [2] : dans ce dernier cas une impureté isomorphe stabilise quelque temps cette phase instable.

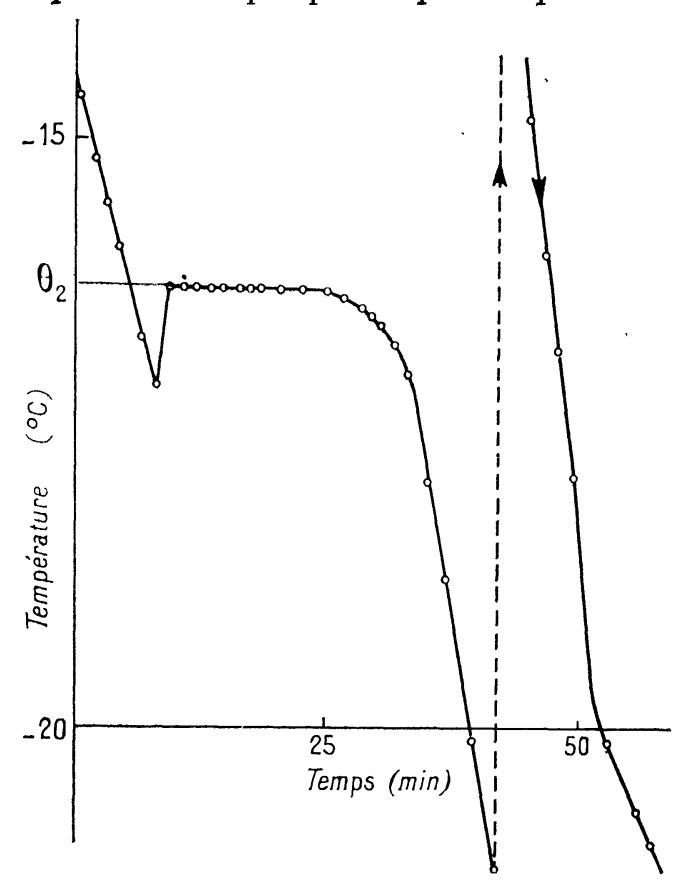

FIg. 2.

La température $\theta_{2}$ est $-16,3 \pm 0,2{ }^{\circ} \mathrm{C}$ : elle correspond à la température d'équilibre entre le liquide et le gallium II.

Des observations aux rayons $\mathrm{X}$ effectuées au laboratoire de minéralogie de $M$. Wyart ont montré l'existence d'une phase solide se formant à la température $\theta_{2}$ du palier de la courbe de refroidissement. Le rapport des conductibilités électriques du liquide et du solide est de l'ordre de $1 / 2,6$, montrant que la phase instable doit avoir une structure plus compacte que celle de la phase stable. L'anisotropie électrique, si prononcée pour le gallium solide stable, semble ne plus subsister.

Pour mesurer la chaleur latente de fusion $L$ de la phase instable, nous avons utilisé une méthode calorimétrique du type électrique [3]. Nous avons trouvé $L=9,09 \pm 0,03 \mathrm{cal} / \mathrm{g}$ : cette valeur correspond à la moitié de la chaleur latente de fusion de la phase stable $(19,14 \mathrm{cal} / \mathrm{g})$. En utilisant les paramètres définissant le point triple $\left(12050 \mathrm{~kg} / \mathrm{cm}^{2}\right.$ et $+2,4^{\circ} \mathrm{C}$ ) la formule de Clapeyron donne une chaleur latente de fusion de $10,3 \mathrm{cal} / \mathrm{g}$.

La transformation de la phase solide instable en phase solide stable se fait avec une forte dilatation que nous avons mesurée au moyen d'un dilatomètre à alcool [2]. $\mathrm{A}-22^{\circ} \mathrm{C}$, les masses spécifiques respectives des phases solide stable, solide instable et liquide sont : $5,92 \mathrm{~g} / \mathrm{cm}^{3}, 6,23 \mathrm{~g} / \mathrm{cm}^{3}$ [2] et $6,135 \mathrm{~g} / \mathrm{cm}^{3}$; celles du solide stable et du liquide sunt en accord avec les données de la littérature [4], [5], [6]. 